\title{
Solving the Mystery of Crypto's Bubble
}

\author{
Harsh Sengar*
}

\section{Abstract}

Blockchain is the vehicle on which cryptocurrencies run, and it can't be regulated by any legal entity during its operation. The enormous growth in various cryptocurrency segments in the last decade has created an unwelcome controversy of an inevitable bubble. Bubble signifies a sudden, significant change of valuation of any asset from its intrinsic valuation. That change may be sustainable for a decent length of time, in case of really large bubbles. A bubble can be generated either by queer herd behaviour or logical secular movement. Traces of evident bubbles have been a certainty, moreover, they take the perceived valuation of crypto to dizzy heights, far away from its true valuation. This sudden diversion can be lethal due to the illogical, irrational propensity of regular market participants. This study observes ten cryptos under surveillance from September 2014 to August 2019. The selected ten (namely Monero, Bitcoin, XRP Ripple, Litecoin, Dogecoin, Monacoin, Ethereum, Bytecoin, Digibite, Potcoin) cryptocurrencies were studied for the last five years using Right Tailed ADF Test. Prominent traces of the rational bubble in all the underlying cryptocurrencies were found and have been considered for our study. The study hopes to create financial prudence among average crypto investors that refrains them from asset classes of high volatility and absent regulation.

Keywords: Cryptocurrency, Bubble, Right Tailed Augmented Dickey Fuller Test, Long range dependence

* Asst. Manager, Financial Control, Barbeque Nation Hospitality Ltd, India; harshsengar1996@gmail.com 


\section{Introduction}

It is human nature to get work done as fast as possible with the desired results and accuracy, which leads to new inventions daily that result in making human life easy. From some decades finance sectors saw great changes which further boosting the economies of the world, the biggest change in finance is block-chain and cryptocurrencies which now making the financial transactions much easier than earlier. We all know that crypto-currency generated to make payment of illegal things earlier in the USA with a motive of un-traceability of the transactions and deals which promote the business of dark web services which not only include smuggling but also includes payments for killing someone, illegal political activities, murders, money laundering, insider trading, etc. But later due to its anonymity it is found to be safer and better for banking transactions while transacting in a different currency as it will be much easier and cheap (no tax). This will not only make transactions safe with its anonymity but fast and as cryptocurrencies are not owned by any government, there is no transaction fee/duty to be levied. So for the future, it will make banking transactions cheaper, faster and safer.

As of July 2018, there are nearly 2000 different cryptocurrencies, some of them like Dogecoin were created only to make fun of the hype on the blockchain based currencies. They may differ in many ways, but all of them have the same core, the blockchain. Broadly speaking, it is a chain of solved crypto-logical codes, so-called proofs of work. According to a study of finance website Finder.com around 8\% Americans owned bitcoin in March 2018.

Cryptocurrencies grabbed the attention of many people, some of them became avid fans of this monetary innovation, while others were much more sceptical. Just like it was in the case of governments, for a few banned in their countries the use of virtual money, while for example, Venezuela issued its own cryptocurrency in an attempt to overcome its economic difficulties.

The total market cap in July 2018 was nearly 300 billion USD, however, only a minority of coins is important on the market, because only $1 \%$ of them constitutes as much as $88 \%$ of the whole market value. The most important is the oldest coin, the bitcoin. It 
is virtual money created in 2009 by mysterious Satoshi Nakamoto, as an alternative monetary unit which would not hold drawbacks of contemporary fiat money, like inflation or necessity of the intermediary - banks.

The main motive of this financial invention is untraceable safe transactions which will decrease the pace of supply and this achieved with the crytological code which is not easy to decode. For this new currency, there is a need for proper network and channel which created by many tech scientists for the incentives of transaction fee and the possibility of mining new coins.

The different benefits of cryptocurrencies make it popular in no time after the financial collapse of 2008 where this seems like an alternate to park money with potentially high interest. This belief and trust about crypto-currencies look fair if we track Bitcoin which is the largest cryptocurrency by market capitalization, has risen over $100,000,000 \%$ in value between its creation in January 2009 and August 2017; till December 2019 the return on investment found as $5298 \%$ if anyone invested at its launch1. Whereas if we look towards Ethereum the second largest cryptocurrency in terms of market capitalization rise $13,728 \%$ in value since 2015 and return on investment is $4344.20 \%$ if anyone invested when it was launched2.

The main question arises when we come to know more about cryptocurrency and dig deep into the question "What makes cryptocurrency so valuable in no time?"

\section{Literature Review}

Monetary policy of the governments impacts the prices of the assets, although it is hard to believe it is not new as incidence proved this phenomenon that how monetary policy and operations of central banks fluctuate prices of the assets. (Sophister, n.d.) This

1 The estimate comes from :

https:/ / coinmarketcap.com/currencies/bitcoin/

2 The estimate comes from :

https://coinmarketcap.com/currencies/ethereum/ 
makes investors find out such securities that get the minimum effect of any government policies. From the words of Rothenberg \& Magalhães, (2013) and Nakamoto (2008) term bitcoin as pure peer to peer electronic transaction which is done through online mode which enables the people to send money from one party to another without going through a financial institution. 'Satoshi Nakamoto3'"Governments are good at cutting off the heads of a centrally controlled network like Napster, but pure P2P networks like Gnutella and Tor seem to be holding their own." Thus this un-traceability feature of cryptocurrency makes it more popular which explained by Hadas (2013) in his article. He mentioned that although Bitcoin is created for better financial transactions, which also maintain security through anonymity, transparency and integrity; there is other factor which makes it to be banned by government is tax evasion which makes it the most disruptive model of financial innovation. The other crimes can be traced now with the help of transaction history, but cannot be traced if payment made in bitcoins.

Thus this type of ambiguity to park untraceable money in cryptocurrency creates the herd behaviour which is a general tendency of human beings that led to tremendous growth in the cryptocurrency. Nakamoto (2008) termed bitcoin as a pure peer to peer electronic transaction that is done through online modes and enables people to send money from one party to another without going through a financial institution. Glaser, Zimmermann, Haferkorn, Weber, \& Siering (2014) in their research paper showed that how in a short duration of time cryptocurrency got popularised as an asset from digital currency and how the acceptability of cryptocurrency as investment raised from dark web users to investors and the general public. Garcia, Tessone, Mavrodiev, \& Perony (2014) in his research proved that how social media creates the word of mouth effect which further increased the trading of cryptocurrency and made it popular in 2009-2012.

3 Satoshi Nakamoto is the name used by the pseudonymous person or persons who developed bitcoin, authored the bitcoin white paper, and created and deployed bitcoin's original reference implementation. As part of the implementation, Nakamoto also devised the first blockchain database. In the process, Nakamoto was the first to solve the doublespending problem for digital currency using a peer-to-peernetwork. 
Another study of Garcia, Mavrodiev, \& Schweitzer (2013) stated that with the study of k-core analysis researchers were able to find out the methods of predicting the upcoming trend of popularity of any underlying assets. Ghosh (2016) in his study on CNX Nifty for the period of $17^{\text {th }}$ September 2007 to $28^{\text {th }}$ April 2016 with ADF, RADF, SADF, and GSADF proved that how an asset gets extremely perceived at a higher value for a short time due to herding behaviour and once people noted its true inherent value it collapsed within a short span of time. In another research by Edmund \& Lindgren (2017), it is found that the herding behaviour of humans led to the popularity of cryptocurrency especially bitcoin which gives extraordinary returns over the traditional investments. Bitcoin and Ether who are highly traded cryptocurrencies and qualified as commodities not subject to any securities laws. Cermak (2017) in his research paper proved that bitcoin is more volatile than any other fiat currency. But if we introduce derivative trading in cryptocurrency where anyone short sell or hedge their position through future contracts will surely limit the volatility of the cryptocurrency.

In the study by Bianchetti, Ricci, \& Scaringi (2017), they proved that cryptocurrency got popular after the 2008 crisis and with the use of peer to peer exchange it got massive. The acceptance of cryptocurrency is increasing day by day but there is no real authority behind it. It is found that how this herd behaviour makes the bubble vulnerable and increased its effect, this was proved by the study of Sornette (1987) that how in 1987, 2000 and even in 2002 market crash became much larger due to the herd behaviour. Ghosh, M.C., Rao, Kozarević, \& Pandey (2018) in their paper show the predictability and herding behaviour in the CNX NIFTY regular as well as CNX NIFTY High Frequency Trading domains with Hurst exponent prove that in both there is significant herd behaviour and it is more predictable as well.

This herd behaviour leads market to collapse with a major crash and economic recession which is nothing but a correction in the market of prices of securities which get perceived much higher than its real intrinsic value. This proved by Dutch Tulip-mania ("The Dutch Tulip Mania: The Social Foundations of a Financial Bubble A. Maurits van der Veen," 2012) where it is clearly 
explained that how in 1636-1637 Tulip perceived price increase and formed a bubble which worth after 8 years was almost $1 / 10$ of the price. With the help of Metcalf's law, Wheatley, Sornette, Huber, Reppen, \& Gantner (2018) explained that whenever a perceived value is higher than fundamental value and people are paying more than fundamental value there is the existence of a bubble and this bubble could burst any time. Ghosh \& Kozarević, (2019) in their research paper with Artificial Neural Network and Multiple Adaptive Regression Splines on the NIFTY data showed the escape velocity in the Indian stock market where bubble breach the threshold and go above like avalanche. Moreover, any assets that have built up spurting over a short period and developed into a vicious bubble ideally percolates 'fear' and display 'power law' behaviour virulently. (Ghosh \& Krishna, 2019; Ghosh, Roux, \& Ianole, 2017)

There is the value of every commodity and security which can be measure with different methods, makes us unable to know the true intrinsic value of the security. In the study by Kuo, Lee, \& Wang (2018) it is discussed that it is really difficult to calculate the real value of cryptocurrency as it does not have any underlying real assets like any other alternative investment classes but their store of value can be related with the development of the technology underneath which is blockchain and the computing power of the mining. Petrov \& Golub (2018) in their research paper proved that volatility in cryptocurrencies is driven by several transactions, he also found that there are particular periods or seasonality that exist in the bitcoin and altcoins when its price is found to be more. While Aalborg, Molnár, \& de Vries (2019) in their research proved that traded volume, transaction volume, return and goggle search are the main explanatory variable which means these variables make a significant impact on prices of bitcoin.

But it is clear that although some factors prove the price we can't ignore the price bubble in the cryptocurrencies. In the research paper of Pástor \& Veronesi (2006) it is proved that how bubble gets formed and bursts when it is evident by the general public. In the recent study by Kristoufek (2014), it is found that how different factors or drivers evolve the bitcoin and in just 30 months bitcoin went up by $1200 \%$, but it is also proved that bitcoin prices corrected 
at different period and never return to the price of the pre-bubble period, in the study the price pattern was able to show the inherent bubble in the bitcoin. In study by Smith, Suchanek, \& Williams (2009) it is found that there is a particular pattern of bubble even if the effect is low which makes investors find out early and can save their money.

For predicting bubble many researchers used different techniques and proved their works like Schaller \& van Norden (2002) used the switching regression to distinguish between competing models for tests the bubble in an inherent asset. The authors proved that there is a bubble in the model. Chong \& Hurn (2016) in their research paper for detecting real time bubble in asset pricing focussed on the recursive and rolling-recursive regressions with the right-tailed unit root tests. He successfully proved the efficiency of rollingrecursive window tests (SADF, GSADF) with the sample data of NASDAQ stock index and also with US housing on the ratio of price to rent where he found the price bubble in both. Fantazzini, Nigmatullin, Sukhanovskaya, \& Ivliev (2017) in his article highlighted the advantages and limits of bitcoin with a review of econometrics and mathematical tools which were used to find out the bitcoin price and several related issues.

Caspi (2017) in his research paper proved and introduce the Rtadf (Right tailed Augmented Dickey Fuller) test in Eviews in 2000 for assets price bubble detection strategy, this is an updated version of ADF unit root test. Catania, Grassi, \& Ravazzolo (2018) in their paper with the help of GARCH model proved the conditional volatility in Bitcoin, Ethereum, Litecoin, and Ripple. In the master thesis of Bern \& Wiedmer (2018) with the unit root co-integration test and granger causality test, they confirmed the bubble in different cryptocurrencies. While Engle, Giglio, Lee, Kelly, \& Stroebel (2019) in their paper with the help of augmented dickey fuller test found out the inherent risk created out of the bubble. After that with the help of the granger causality test they tried to find out if it is the hype driving the bitcoin or bitcoin driving the hype, later they conclude that the price of bitcoin drives the hype which making its popular day by day. Mitra \& Choudhuri (2016) uses three variants of the right tailed augmented dickey fuller tests (ADF, SADF and RADF) on BSE Sensex data for 2008 global crises 
till 2014 and conclude that there is no inherent bubble in it but they also mention about the reason of inherent bubble is herding behaviour of the general public who don't take decision rationally. In the study of Phillips (2015) where on the basis of PSY, PWY and sequential PWY algorithm he found out the multiple bubbles and give results accordingly and justifies the use of the algorithmic model in bubble testing. The research of Kroll (2019) with the help of GARCH Model proved that from 2011 to 2018 bitcoin was not found efficient but was rather found to be in the speculative stage. There is market inefficiency in the bitcoin and bitcoin also proved that there is no exposure to traditional risks like interest rate risk, market risk or currency risk on it.

\section{Research Methodology}

$\gamma(t)=\mu+\delta \gamma(t-1)+\sum_{i=1}^{\rho} \cdot \varphi i \Delta \gamma(t-i)+\varepsilon(t)$

$\gamma(t)$ here is the crypto-currencies' daily closing price, $\mu$ is intercept, $\varphi$ is the coefficient for differentiated $i$ lags, whereas $\mathrm{p}$ is the maximum numbers of lags and $\varepsilon$ is the error. For the study, we choose ten different cryptocurrencies and conduct our research by breaking them with a different period to understand them properly.

The cryptocurrency is choosen on the basis of popularity, time period, and volume thus we choose Bitcoin, Bytecoin, Mona Coin, Potcoin, Digibyte, Dash Coin, XRP, Dogecoin, Monero and Litecoin; with sample of 1887 of every cryptocurrency. Augmented Dickey Fuller test at different points of lag is conducted on these selected crypto-currencies' daily closing between 21 March 2014 to 19 July 2019. For better understanding and clear picture, we break these data of each crypto in five different years. Thus, every sub sample is finite and fixed in nature.

\subsection{Hypothesis setting}

The null (Ho) and alternative (Ha) hypothesis may be written as:

Ho: $\delta=1$

Ha: $\delta>1$ 
where, Ho indicates that the time series has a unit root, so it is nonstationary and, Ha indicates that time series has not any unit root and stationery in nature which means that it has explosive autoregressive coefficients.

Thus, if P-value is less than 5\%, Ho is rejected and we can conclude that evidence of a price bubble will be there. On the other side if the value of $\mathrm{P}$ is more than $5 \%$, then Ho is accepted and it concludes the absence of any price bubble.

\subsection{Interpretation of the result}

The Right tailed Augmented Dickey Fuller Test conducted for these ten cryptocurrencies and the data includes the total 150 tests (Sengar, 2020)4. In all the 150 tests consists of level, $1^{\text {st }}$ difference and $2^{\text {nd }}$ difference we find that there is the existence of an explosive bubble in the Bitcoin and Altcoin.

We selected these cryptocurrencies on the basis of the availability of data as well as their circulating supply limit.

Monero: The open-source cryptocurrency which is created in April 2014 with ticker symbol XMR having circulating supply of 17,066,158 (as of June 2019). Monero got popular after its introduction of the Ring Confidential Transaction system.

Bitcoin: Bitcoin is the most expensive and popular cryptocurrency in the world. The developer of Bitcoin is still unknown whereas the name used in 2008 for development is Satoshi Nakamoto. The main motive behind the invention of Bitcoin is to support and to make payment in the dark/deep web.

Litecoin: Litecoin was released via an open source client on GitHub on October 7, 2011 by Charlie Lee. The supply limit of litecoin is 84,000,000 LTC with ticker symbol L.

Dogecoin: Dogecoin was released on December 6, 2013 by Billy Markus and Jackson Palmer. As of February 2018, over 113 billion coins had been mined.

4 This file contains the Tests result which is in 150 pictures. 
XRP of Ripple: XRP was released via an open source client on GitHub in 2012 by Arthur Britto, David Schwartz and Ryan Fugger. As of September 2019, over 100 billion coins had been mined yet and supplied.

Dashcoin: Dashcoin was released on January 18, 2014 by Evan Duffield. As of September 2019, over 17,574,970 DSH coins have in circulation.

Bytecoin: Bytecoin was released via an open source client on GitHub on October 7,2011 by Charlie Lee. The supply limit of bytecoin is 84,000,000 LTC with ticker symbol L.

Digicoin: Digicoin was developed by Andrew Davidson on May 20, 2013 which also known as Securecoin and Argentum. The supply limit of Digicoin is 48.17 million DGC.

Monacoin: Monacoin was developed in January 2013 on the forum named Mr. Watanabe. The supply limit of Monacoin is 105.12 million DGC.

Potcoin: Potcoin was developed by Hasoshi, Mr. Jones and Smokemon 514 in the month of January of 2013. The available supply of Potcoin is 420 million.

\section{Results of Tests}

\begin{tabular}{cccccccc}
\hline Crypto & Years & \multicolumn{2}{c}{ Level } & \multicolumn{2}{c}{$\mathbf{1}^{\text {st }}$ Difference } & \multicolumn{2}{c}{$\begin{array}{c}2^{\text {nd }} \\
\text { Difference }\end{array}$} \\
\hline \multirow{6}{*}{ Monero } & & t- stat & $\begin{array}{c}\text { p- } \\
\text { value }\end{array}$ & t- stat & $\begin{array}{c}\text { p- } \\
\text { value }\end{array}$ & t- stat & $\begin{array}{c}\text { p- } \\
\text { val } \\
\text { ue }\end{array}$ \\
\hline & $2014-15$ & -16.56 & $0 \%$ & -13.92 & $0 \%$ & -12.447 & $0 \%$ \\
& $2015-16$ & -21.1159 & $0 \%$ & -11.92 & $0 \%$ & -12.27 & $0 \%$ \\
& $2016-17$ & -16.68 & $0 \%$ & -12.84 & $0 \%$ & -11.26 & $0 \%$ \\
& $2017-18$ & -21.64 & $0 \%$ & -17.68 & $0 \%$ & -12.188 & $0 \%$ \\
& $2018-19$ & -22.56 & $0 \%$ & -16.205 & $0 \%$ & -11.038 & $0 \%$ \\
Bitcoin & $2015-16$ & -10.28 & $0 \%$ & -15.87 & $0 \%$ & -13.82 & $0 \%$ \\
& $2016-17$ & -18.182 & $0 \%$ & -15.952 & $0 \%$ & -15.93 & $0 \%$ \\
& $2017-18$ & -18.766 & $0 \%$ & -11.562 & $0 \%$ & -11.537 & $0 \%$ \\
& $2018-19$ & -22.298 & $0 \%$ & -11.064 & $0 \%$ & -12.97 & $0 \%$
\end{tabular}




\begin{tabular}{|c|c|c|c|c|c|c|c|}
\hline \multirow{5}{*}{ Litecoin } & 2014-15 & -14.67 & $0 \%$ & -11.36 & $0 \%$ & -13.70 & $0 \%$ \\
\hline & $2015-16$ & -15.87 & $0 \%$ & -15.77 & $0 \%$ & -11.58 & $0 \%$ \\
\hline & $2016-17$ & -18.97 & $0 \%$ & -15.75 & $0 \%$ & -11.07 & $0 \%$ \\
\hline & 2017-18 & -18.57 & $0 \%$ & -16.39 & $0 \%$ & -13.32 & $0 \%$ \\
\hline & 2018-19 & -21.06 & $0 \%$ & -11.17 & $0 \%$ & -11.36 & $0 \%$ \\
\hline \multirow{5}{*}{ Dogecoin } & 2014-15 & -19.93 & $0 \%$ & -11.88 & $0 \%$ & -12.34 & $0 \%$ \\
\hline & $2015-16$ & -17 & $0 \%$ & -12.203 & $0 \%$ & -10.796 & $0 \%$ \\
\hline & 2016-17 & -18.845 & $0 \%$ & -11.33 & $0 \%$ & -10.98 & $0 \%$ \\
\hline & 2017-18 & -19.62 & $0 \%$ & -14.298 & $0 \%$ & -14.45 & $0 \%$ \\
\hline & 2018-19 & -17.51 & $0 \%$ & -14.04 & $0 \%$ & -11.562 & $0 \%$ \\
\hline \multirow{5}{*}{$\begin{array}{l}\text { XRP of } \\
\text { Ripple }\end{array}$} & 2014-15 & -16.185 & $0 \%$ & -14.096 & $0 \%$ & -10.928 & $\%$ \\
\hline & $2015-16$ & -15.459 & $0 \%$ & -19.762 & $0 \%$ & -11.73 & $0 \%$ \\
\hline & 2016-17 & -12.055 & $0 \%$ & -12.99 & $0 \%$ & -10.78 & $\%$ \\
\hline & 2017-18 & -18.23 & $0 \%$ & -12.86 & $0 \%$ & -10.53 & $0 \%$ \\
\hline & 2018-19 & -20.49 & $0 \%$ & -12.91 & $0 \%$ & -12.52 & $\%$ \\
\hline \multirow{5}{*}{ DashCoin } & 2014-15 & -19.51 & $0 \%$ & -12.79 & $0 \%$ & -10.77 & $\%$ \\
\hline & $2015-16$ & -21.88 & $0 \%$ & -14.61 & $0 \%$ & -11.53 & $\%$ \\
\hline & 2016-17 & -18.07 & $0 \%$ & -13.44 & $0 \%$ & -10.65 & $\%$ \\
\hline & 2017-18 & -20.86 & $0 \%$ & -13.16 & $0 \%$ & -12.35 & $0 \%$ \\
\hline & 2018-19 & -21.97 & $0 \%$ & -15.37 & $0 \%$ & -12.66 & $\%$ \\
\hline \multirow{5}{*}{ Bytecoin } & 2014-15 & -22.69 & $0 \%$ & -13.46 & $0 \%$ & -12.68 & $0 \%$ \\
\hline & $2015-16$ & -22.50 & $0 \%$ & -11.31 & $0 \%$ & -10.799 & $0 \%$ \\
\hline & 2016-17 & -24.03 & $0 \%$ & -11.28 & $0 \%$ & -11.45 & $0 \%$ \\
\hline & 2017-18 & -24.05 & $0 \%$ & -13 & $0 \%$ & -11.19 & $0 \%$ \\
\hline & 2018-19 & -23.56 & $0 \%$ & -11.78 & $0 \%$ & -12.55 & $0 \%$ \\
\hline \multirow{5}{*}{ Digicoin } & 2014-15 & -20.16 & $0 \%$ & -15.33 & $0 \%$ & -10.56 & $0 \%$ \\
\hline & $2015-16$ & -18.35 & $0 \%$ & -12.57 & $0 \%$ & -14.05 & $0 \%$ \\
\hline & 2016-17 & -18.77 & $0 \%$ & -10.96 & $0 \%$ & -11.39 & $0 \%$ \\
\hline & 2017-18 & -19.72 & $0 \%$ & -15.06 & $0 \%$ & -10.67 & $0 \%$ \\
\hline & 2018-19 & -22.62 & $0 \%$ & -17.17 & $0 \%$ & -12.06 & $0 \%$ \\
\hline \multirow{5}{*}{ Monacoin } & 2014-15 & -18.76 & $0 \%$ & -14.63 & $0 \%$ & -12.76 & $0 \%$ \\
\hline & $2015-16$ & -21.43 & $0 \%$ & -15.304 & $0 \%$ & -11.56 & $0 \%$ \\
\hline & 2016-17 & -20.202 & $0 \%$ & -12.302 & $0 \%$ & -10.625 & $0 \%$ \\
\hline & 2017-18 & -16.58 & $0 \%$ & -15.02 & $0 \%$ & -11.74 & $0 \%$ \\
\hline & 2018-19 & -20.496 & $0 \%$ & -13.84 & $0 \%$ & -10.99 & $0 \%$ \\
\hline \multirow{3}{*}{ Potcoin } & 2014-15 & -20.35 & $0 \%$ & -12.199 & $0 \%$ & -12.344 & $0 \%$ \\
\hline & $2015-16$ & -23.54 & $0 \%$ & -14.22 & $0 \%$ & -12.96 & $0 \%$ \\
\hline & 2016-17 & -19.87 & $0 \%$ & -12.78 & $0 \%$ & -11.81 & 0 \\
\hline
\end{tabular}




\begin{tabular}{lllllll}
$2017-18$ & -20.62 & $0 \%$ & -13.86 & $0 \%$ & -12.25 & $0 \%$ \\
$2018-19$ & -23.56 & $0 \%$ & -13.04 & $0 \%$ & -12.16 & $0 \%$ \\
\hline
\end{tabular}

\section{Conclusion}

The main motive of this research paper was to find out the general mechanism which triggers speculative bubbles off. In this attempt to get clarity and removing the curtain from the cryptocurrencies' role I follow the method of Itamar Caspi which includes the use of right tailed Augmented Dickey Fuller Test in the Econometrics software Eviews. Most of the literature also indicates the efficiency of right tailed test method for finding out the speculations in intrinsic price which is also called a bubble. Based on the study we can conclude that all the cryptocurrencies (Monero, Bitcoin, Litecoin, Dogecoin, XRP of Ripple, Dashcoin, Bytecoin, Digicoin, Monacoin and Potcoin) have an inherent bubble in its value and at each time lag which is proved with the help of the analysis from Augmented Dickey Fuller Test. The perceived value found more of Bitcoin, Bytecoin, Mona Coin, Potcoin, Digibyte, Dash Coin, XRP, Dogecoin, Monero and Litecoin whereas the fundamental value is still not that much. The main thing which creates the bubble in this cryptocurrencies is the un-traceability of illegal or any transactions, herd behaviour, and social media influence. But we can't neglect the volume of transactions that happened in Cryptocurrencies as well as mining and development of blockchain technology which creates and develop the value of cryptocurrencies.

There is an extreme need for research on topics like this now as we are forwarding toward a digital economy where we try to make everything possible digitally like digital payments, transactions, payments of duty, banking, shopping etc. We are forwarding toward it with the help of block-chain which majorly depends on cryptocurrency but if the foundation is itself shaky the how we can proceed towards it.

Thus further research is needed and it should be done in this domain of blockchain based currencies as it is still novel and unexplained subject, therefore, checking of the bubble if any in the 
bitcoin or the altcoin 5 is always required. Moreover, this model can also further be developed and used for the research in this domain.

\section{References}

Aalborg, H. A., Molnár, P., \& de Vries, J. E. (2019). What can explain the price, volatility and trading volume of Bitcoin? Finance Research Letters, 29, 255-265.

Bern, U., \& Wiedmer, J. (2018). The Price of Cryptocurrencies: An Empirical Analysis Chair of Economic Theory.

Bianchetti, M., Ricci, C., \& Scaringi, M. (2017). Are Cryptocurrencies Real Financial Bubbles? Evidence from Quantitative Analyses. Ssrn, 1-13.

Caspi, I. (2017). RTADF Testing for Bubbles with EViews. Journal of Statistical Software, 81(1), 1-16.

Catania, L., Grassi, S., \& Ravazzolo, F. (2018). Predicting the Volatility of Cryptocurrency Time-Series. Mathematical and Statistical Methods for Actuarial Sciences and Finance, (3), 203-207.

Cermak, V. (2017). Can Bitcoin Become a Viable Alternative to Fiat Currencies? An empirical analysis of Bitcoin 's volatility based on a GARCH model.

Chong, J., \& Hurn, A. S. (2016). Testing for Speculative Bubbles: Revisiting the Rolling Window.

Engle, R., Giglio, S., Lee, H., Kelly, B., \& Stroebel, J. (2019). CESifo Working Paper no. 7655.

Fantazzini, D., Nigmatullin, E., Sukhanovskaya, V., \& Ivliev, S. (2017). Everything you always wanted to know about bitcoin modeling but were afraid to ask. Part 2. Applied Econometrics, 45, 5-28.

Garcia, D., Mavrodiev, P., \& Schweitzer, F. (2013). Social Resilience in Online Communities : The Autopsy of Friendster, 1-22.

Garcia, D., Tessone, C. J., Mavrodiev, P., \& Perony, N. (2014). The digital traces of bubbles: Feedback cycles between socio-economic signals in the Bitcoin economy. Journal of the Royal Society Interface, 11(99).

Ghosh, B. (2016). Rational Bubble Testing: An in-depth Study on CNX Nifty. Asian Journal of Research in Banking and Finance, 6(6), 10-16.

Ghosh, B., \& Kozarević, E. (2019). Escape velocity backed avalanche predictor neural evidence from nifty. International Journal of Recent Technology and Engineering, 8(4), 486-490.

Ghosh, B., \& Krishna, M. C. (2019). Power law in tails of bourse volatility evidence from India. Investment Management and Financial Innovations,

5 The term used for the alternative of bitcoin which includes every cryptocurrencies except bitcoin. 
16(1), 291-298.

Ghosh, B., M.C., K., Rao, S., Kozarević, E., \& Pandey, R. K. (2018). Predictability and herding of bourse volatility: an econophysics analogue. Investment Management and Financial Innovations, 15(2), 12.

Ghosh, B., Roux, C. Le, \& Ianole, R. (2017). Fear estimation-evidence from BRICS and UK. International Journal of Applied Business and Economic Research, 15(4), 15.

Glaser, F., Zimmermann, K., Haferkorn, M., Weber, M. C., \& Siering, M. (2014). Bitcoin - Asset or currency? Revealing users' hidden intentions. ECIS 2014 Proceedings - 22nd European Conference on Information Systems, 1-14.

Hadas, E. (2013). A Prediction: Bitcoin Is Doomed to Fail.

Kristoufek, L. (2014). What are the main drivers of the Bitcoin price? Evidence from wavelet coherence analysis, (23).

Kroll, S. (2019). Thesis Bitcoin Price Formation: An Empirical Investigation Submitted By Aric Light Department of Economics In partial fulfillment of the requirements For the Degree of Master of Arts Colorado State University Fort Collins, Colorado Summer 2019 Master' .

Kuo, D., Lee, C., \& Wang, Y. (2018). Cryptocurrency: A new investment opportunity?, 16-40.

Mitra, A., \& Choudhuri, S. A Study on Rational Price Bubble in S\&P Bse Sensex. International Journal of Business Quantitative Economics and Applied Management Research, 3(4), 13-19.

Mokhtarian, E., \& Lindgren, A. (2017). Rise of the Crypto Hedge Fund: Operational Issues and Best Practices for Emergent Investment Industry. Stanford Journal of Law, Business, and Finance, Forthcoming.

Nakamoto, S. (2008). Bitcoin: A Peer-to-Perr Electornic Cash System.

Pástor, L'., \& Veronesi, P. (2006). Was there a Nasdaq bubble in the late 1990s? Journal of Financial Economics, 81(1), 61-100.

Petrov, V., \& Golub, A. (2018). Volatility seasonality of Bitcoin prices, i, 14.

Phillips, P. C. B. (2015). Testing for Multiple Bubbles: Limit Theory of Real- time Detectors Testing for Multiple Bubbles 2 : Limit Theory of Real Time Detectors, 1079-1134.

Rothenberg, C. E., \& Magalhães, M. F. (2013). Bitcoin A Peer-to-Peer Electronic Cash System.

Schaller, H., \& van Norden, S. (2002). Fads or bubbles? Empirical Economics, 27(2), 335-362.

Sengar, H. (2020). Annexure MT.

Smith, V. L., Suchanek, G. L., \& Williams, A. W. (1988). Bubbles, crashes, and endogenous expectations in experimental spot asset markets. Econometrica: Journal of the Econometric Society, 1119-1151. 
Sophister, S. (n.d.). Should Central Banks Burst asset Price Bubbles?, 3246.

Sornette, D. (1987). Why Stock Markets Crash.

The Dutch Tulip Mania: The Social Foundations of a Financial Bubble A. Maurits van der Veen. (2012).

Wheatley, S., Sornette, D., Huber, T., Reppen, M., \& Gantner, R. N. (2018). Are Bitcoin Bubbles Predictable? Combining a Generalized Metcalfe's Law and the LPPLS Model. Ssrn. 